\title{
Parecer BRASPEN/AMIB para o enfrentamento da COVID-19 em pacientes hospitalizados
}

\section{BRASPENIAMIB opinion for coping with COVID-19 in hospitalized patients}

\author{
Apoio institucional da Associação de Medicina Intensiva Brasileira (AMIB)
}

\section{DOI: $10.37111 /$ braspenj.parecerbraspen2020}

\section{Letícia Fuganti Campos'}

Priscilla Alves Barreto ${ }^{2}$

Guilherme Duprat Ceniccola ${ }^{3}$

Rodrigo Costa Gonçalves ${ }^{4}$

Clarissa Martins Saraiva Figueira Zambelli

Melina Gouveia Castro ${ }^{7}$
Liane Brescovici Nunes de Matos ${ }^{5}$

O combate à pandemia da COVID-19 se tornou o grande desafio atual, e a terapia nutricional é parte fundamental do cuidado integral na atenção ao paciente crítico. A maioria dos pacientes contaminados tem sido tratada em casa, em isolamento domiciliar. Entretanto, uma parte destes pacientes complica e necessita hospitalização, e cerca de $5 \%$ precisam de terapia intensiva. Neste subgrupo, as complicações mais frequentes são a disfunção respiratória, seguida da disfunção renal.

Sendo assim, BRASPEN/AMIB divulgam, a seguir, sugestões para orientar as equipes multidisciplinares de terapia nutricional (EMTNs) no nosso País:

\section{ORIENTAÇÕES QUANTO À TERAPIA NUTRICIONAL}

\section{Unitermos:}

Coronavirus. Terapia nutricional. Pacientes internados.

\section{Keywords:}

Coronavirus. Nutrition therapy. Inpatients.

\section{Endereço para correspondência:} Melina Gouveia Castro

Rua Abilio Soares, 233 - ci 144 - Paraíso

São Paulo - SP - CEP: 04005-000

E-mail: melinacastro@globo.com

\section{Submissão}

14 de março de 2020

Aceito para publicação

23 de março de 2020

\section{Risco nutricional do paciente com COVID-19}

Alinhados com diversas diretrizes de terapia nutricional (TN), recomenda-se a realização da triagem nutricional em até 48 horas após a admissão hospitalar, em todos os pacientes. Considerando a pandemia atual da COVID-19, recomendamos, entretanto, evitar o contato físico do nutricionista com os pacientes, conforme detalhamos nas sugestões de mudança de rotina.

Sugerimos que, durante a internação dos pacientes com COVID-19, sejam incluídas perguntas para triagem nutricional, segundo protocolo estabelecido pela instituição, sempre que possível.

Os pacientes que permanecerem por mais de 48 horas na unidade de terapia intensiva (UTI) devem ser considerados em risco de desnutrição. Conforme a recomendação da ESPEN 2019, este paciente se beneficia de TN precoce e individualizada.

1. Presidente do Comitê de Nutrição da Sociedade Brasileira de Nutrição Parenteral e Enteral (BRASPEN). Doutora em Clínica Cirúrgica pela Universidade Federal do Paraná (UFPR). Mestre pela Faculdade de Medicina da Universidade de São Paulo (USP). Especialista em Nutrição Clínica pelo GANEP e em Educação em Diabetes pela UNIP. Membro do Departamento de Nutrição da Sociedade Brasileira de Diabetes, Curitiba, PR, Brasil.

2. Especialista em Terapia Nutricional - UERJ. Título de especialista em Nutrição Parenteral e Enteral - BRASPEN. Título de especialista em Nutrição Clínica - ASBRAN. Membro do Comitê de Nutrição - BRASPEN. Membro do Comitê de Nutrição - BRASPEN/RJ. Coordenadora do Departamento de Nutrição - SOTIERJ, Rio de Janeiro, RJ, Brasil.

3. Nutricionista do Hospital de Base do D.F. Especialista em nutrição parenteral e enteral pela BRASPEN. Mestre e Doutor em Nutrição Humana pela Universidade de Brasília, Brasília, DF, Brasil.

4. Título de Especialista em Medicina Intensiva, Nutrologia e Nutrição Parenteral e Enteral. Coordenador Clínico EMTN - Hospital HUGOL e Hospital Órion -GO. Presidente Comitê Terapia Nutricional - AMIB, Goiânia, GO, Brasil.

5. Hospital São Luiz Itaim e Vila Nova Star. Medica Intensivista pela AMIB e Especialista em Terapia Nutricional Enteral e Parenteral pela BRASPEN, São Paulo, SP, Brasil.

6. Especialista em Cirurgia Geral com área de atuação em Nutrição Parenteral e Enteral pela BRASPEN. Rede Mater Dei de Saúde. Hospital Luxemburgo, Belo Horizonte, MG, Brasil.

7. Doutora em Cirurgia do Aparelho Digestivo pela Faculdade de Medicina da Universidade de São Paulo. Especialista em Medicina Desportiva pela Universidade Federal de São Paulo. Especialista em Nutrição Clínica pelo Grupo de Nutrição Humana. Especialista em Terapia Nutricional Enteral e Parenteral pela BRASPEN. Presidente da Sociedade Brasileira de Nutrição Enteral e Parenteral -BRASPEN. Médica Nutróloga do departamento de Terapia Nutricional do Hospital Israelita Albert Einstein, São Paulo, SP, Brasil. 


\section{Via de alimentação}

A alimentação por via oral é a preferencial em pacientes não graves com diagnóstico de COVID-19, incluindo a utilização de suplementos orais, quando a ingestão energética estimada for $<60 \%$ das necessidades nutricionais.

Em pacientes graves, a nutrição enteral (NE) é a via preferencial e sugerimos que seja iniciada entre 24 e 48 horas. No caso de contraindicação da via oral e/ou enteral, a nutrição parenteral (NP) deve ser iniciada o mais precocemente possível. Sugerimos considerar o uso de NP suplementar após 5 a 7 dias, em pacientes que não conseguirem atingir aporte calórico proteico $>60 \%$ por via digestiva.

\section{Aporte calórico na fase aguda}

Sugerimos iniciar com aporte calórico mais baixo, entre 15 a $20 \mathrm{kcal} / \mathrm{kg} /$ dia e progredir para $25 \mathrm{kcal} / \mathrm{kg} /$ dia, após o quarto dia dos pacientes em recuperação. Recomenda-se não utilizar calorimetria indireta, pelo risco de disseminação da doença.

Sugerimos utilizar fórmulas enterais com alta densidade calórica $(1,5-2,0 \mathrm{kcal} / \mathrm{ml})$, em pacientes com disfunção respiratória aguda e/ou renal, objetivando restrição da administração de fluidos.

\section{Aporte proteico fase aguda}

Sugerimos que estes doentes recebam entre 1,5 e 2,0 $\mathrm{g} / \mathrm{kg} /$ dia de proteína, mesmo em caso de disfunção renal.

Com a seguinte sugestão de progressão: $<0,8 \mathrm{~g} / \mathrm{kg} / \mathrm{dia}$, no $1^{\circ}$ e $2^{\circ}$ dias, 0,8-1,2 g/ $\mathrm{kg} / \mathrm{dia}$, nos dias 3-5, e > 1,2 g/ $\mathrm{kg} /$ dia, após $\circ 5^{\circ}$ dia.

\section{Fórmula especializada}

Sugerimos não utilizar fórmulas com alto teor lipídico/ baixo teor de carboidrato para manipular coeficiente respiratório e reduzir produção de $\mathrm{CO}_{2}$, em pacientes críticos com disfunção pulmonar.

O uso de uma fórmula enteral com ômega 3, óleos de borragem e antioxidantes em pacientes com síndrome do desconforto respiratório agudo (SDRA) não está indicado.

Sugerimos evitar utilização de módulos pela maior manipulação do paciente e aumento do risco para equipe de enfermagem.

\section{Monitoramento de fósforo sérico}

Sugerimos o monitoramento frequente do fósforo sérico em pacientes críticos e reposição adequada, quando indicada. A hipofosfatemia pode estar sinalizando síndrome de realimentação e a deficiência de fósforo pode contribuir para retardo no desmame ventilatório de pacientes críticos.

Sugerimos que a progressão calórica seja adiada, em pacientes com níveis baixos de baixos de fósforo, potássio ou magnésio, até a correção, com posterior aumento gradual.

\section{TN e Hipoxemia}

Sugerimos que a NE seja mantida em caso de hipercapnia compensada ou permissiva. Suspender a dieta em caso de descompensada hipoxemia, hipercapnia ou acidose grave.

\section{ORIENTAÇÕES PARA O USO DE DIETA ENTERAL EM POSIÇÃO PRONA}

Com a prevalente recomendação da posição prona em pacientes com COVID-19, sugerimos alguns cuidados adicionais com a TN:

- Sugerimos não realizar endoscopia digestiva alta nesta população, pelo alto risco de contaminação;

- Sugerimos que a NE seja continuada durante a posição prona. Destacamos o cuidado em pausar a dieta antes de movimentar o paciente para posição pronada, conforme o tempo sugerido por protocolo local;

- Sugerimos utilizar fórmula hipercalórica hiperproteica sem fibras em volume trófico (até $20 \mathrm{ml} / \mathrm{h}$ ) durante todo o período de prona ou primeiros 6 dias;

- Manter cabeceira elevada em 25-30 (Trendelemburg Reverso);

- Sugerimos prescrever prócinético fixo (metroclopramida ou eritromicina);

- Ofertar NE de maneira contínua, em bomba de infusão;

- Iniciar a dieta após a primeira hora e manter até 1 hora antes do retorno à posição supina.

\section{Se já estiver em uso de TN}

- Sugerimos pausar a dieta enteral e abrir a sonda em sifonagem $2 \mathrm{~h}$ antes da manobra de pronar o paciente e reiniciar a NE 1 h após;

- Sugerimos não suspender nutrição parenteral para execução da manobra.

\section{CONCLUSÃO}

Apesar das dificuldades da pandemia atual, é importante ressaltar que os pacientes acompanhados pela EMTN devem seguir recebendo o suporte nutricional e atenção que necessitam, sem nenhuma repercussão negativa em seu tratamento. 


\section{REFERÊNCIAS}

1. Alhazzani W, Møller MH, Arabi YM, Loeb M, Gong MN, Fan E, et al. Surviving Sepsis Campaign: guidelines on the management of critically ill adults with Coronavirus Disease 2019 (COVID-19). Intensive Care Med. 2020. doi: 10.1007/ s00134-020-06022-5.

2. Reintam Blaser A, Starkopf J, Alhazzani W, Berger MM, Casaer MP, Deane AM, et al.; ESICM Working Group on Gastrointestinal Function. Early enteral nutrition in critically ill patients: ESICM clinical practice guidelines. Intensive Care Med. 2017;43(3):380-98.

3. Castro MG, Ribeiro PC, Souza IAO, Cunha HFR, Silva MHN, Rocha EEM, et al. Diretriz brasileira de terapia nutricional no paciente grave. BRASPEN J. 2018;33(Supl 1):2-36.

4. Silva JSV, Seres DS, Sabino K, Adams SC, Berdahl GJ, Citty SW, et al.; Parenteral Nutrition Safety and Clinical Practice Committees, American Society for Parenteral and Enteral Nutrition. ASPEN Consensus recommendations for refeeding syndrome. Nutr Clin Pract. 2020;35(2):178-95.
5. McClave SA, Taylor BE, Martindale RG, Warren MM, Johnson $\mathrm{DR}$, Braunschweig C, et al. Guidelines for the provision and assessment of nutrition support therapy in the adult critically ill patient: Society of Critical Care Medicine (SCCM) and American Society for Parenteral and Enteral Nutrition (A.S.P.E.N.). JPEN J Parenter Enteral Nutr. 2016;40(2):159-211.

6. Oliveira VM, Piekala DM, Deponti GN, Batista DCR, Minossi $\mathrm{SD}$, Chisté $\mathrm{M}$, et al. Checklist da prona segura: construção e implementação de uma ferramenta para realização da manobra de prona. Rev Bras Ter Intensiva. 2017;29(2):131-41.

7. Conselho Federal de Nutricionistas. Recomendações do CFN boas práticas para a atuação do nutricionista e do técnico em nutrição e dietética durante a pandemia do novo Coronavírus (COVID-19). Brasília: Conselho Federal de Nutricionistas; 2020. [cited 2020 Mar 12]. Available from: https://portaldenutricao.com/wp-content/uploads/2020/03/recomendacoes-cfncoronavirus.pdf

8. Singer P, BlaserAR, Berger MM, Alhazzani W, Calder PC, Casaer MP, et al. ESPEN guideline on clinical nutrition in the intensive care unit. Clin Nutr. 2019;38(1):48-79.

Local de realização do estudo: Sociedade Brasileira de Nutrição Parenteral e Enteral (BRASPEN), São Paulo, SP, Brasil.

Conflito de interesse: Os autores declaram não haver. 